\title{
Localized Amyloidosis of the Upper Urinary Tract: A Case Series of Three Patients Managed with Reconstructive Surgery or Surveillance
}

\author{
Tudor Borza, B.S., Rajal B. Shah, M.D., ${ }^{2}$ Gary J. Faerber, M.D., and J. Stuart Wolf, Jr., M.D.
}

\begin{abstract}
Objective: To evaluate the clinical presentation, diagnosis, treatment, and prognosis of primary localized amyloidosis of the upper urinary tract.

Methods: Patients with primary localized amyloidosis of the upper urinary tract were identified by database inquiry, and their medical records were reviewed.

Results: Primary localized amyloidosis was identified in the ureter in two patients and in the renal pelvis in one patient. Systemic disease and amyloidosis secondary to a medical condition were excluded. All three patients presented with gross painless hematuria, and two also reported flank pain. None endorsed irritative urinary symptoms. Initial evaluation consisted of renal ultrasonography or intravenous urogram, and subsequently additional imaging and ureteroscopies with biopsies were performed. Congo red staining of the biopsies displayed apple green birefringence under polarized light. One patient was treated with distal ureterectomy and Boari flap ureteroneocystostomy, whereas the other two were followed with surveillance imaging. None of the patients have developed progressive disease or recurrence of their symptoms during follow-up of 15 months, 6 years, and 8 years.

Conclusions: Primary localized amyloidosis of the upper urinary tract is a rare condition that is of interest because the clinical presentation and radiographic and endoscopic appearance mimic malignancy. Many commonalities exist between upper urinary tract and bladder amyloidosis, but obstructive features, like flank pain and hydronephrosis, are unique to upper urinary tract lesions. In the absence of significant obstruction, early eradication of an upper urinary tract lesion may not be necessary. Rather, follow-up with serial imaging is sufficient to monitor for disease progression.
\end{abstract}

\section{Introduction}

A MYLOIDOSIS IS A HETEROGENEOUS GROUP of disorders characterized by the extracellular deposition of insoluble fibrillar protein in various organs and tissues. The four major forms of amyloidosis are primary, secondary, heredofamilial, and $\beta_{2}$-microglobulin-associated. Amyloidosis can be further classified as localized, involving a single organ, or systemic, involving multiple organs. Systemic amyloidosis is progressive and usually fatal. In contrast, localized amyloidosis does not progress and is rarely fatal. Most commonly, localized amyloidosis occurs in the respiratory tract, skin, and genitourinary tract. ${ }^{1}$

Localized amyloidosis of the upper urinary tract is a rare condition, as urinary tract amyloidosis is typically confined to the bladder. ${ }^{2}$ Nonetheless, localized amyloidosis of the upper urinary system is of interest because its clinical presentation and radiographic and endoscopic appearance mimic those of upper tract urothelial carcinoma. Accurate diagnosis can avoid nephroureterectomy, which would be the standard treatment for suspected upper tract urothelial carcinoma. We report three cases of localized amyloidosis of the upper urinary tract, two involving the ureters and one involving the renal pelvis.

\section{Methods}

Three patients who were found to have primary localized amyloidosis of the upper urinary tract between 1997 and 2007 were identified by searching the Anatomic Surgery Database at the University of Michigan Hospital. A retrospective chart review was conducted focusing on clinical presentation, investigations, treatment, and follow-up.

Two patients with primary localized amyloidosis of the ureter and one patient with primary localized amyloidosis of the renal pelvis were identified. Patient 1 was a 58-year-old man with amyloidosis of the right renal pelvis. Both patient 2 ,

Departments of ${ }^{1}$ Urology and ${ }^{2}$ Pathology, University of Michigan Health System, Ann Arbor, Michigan. 
a 69-year-old woman, and patient 3, a 37-year-old woman, were found to have amyloidosis of the left ureter. Each of the patients had a single lesion.

\section{Results}

Clinical, radiologic, and laboratory findings (including normal urinary Bence-Jones protein assay, 24-hour urine protein electrophoresis, and serum protein electrophoresis) indicated that none of the patients had primary systemic amyloidosis or amyloidosis secondary to a general medical condition

All three patients presented for care with a single episode of gross, painless hematuria as their initial complaint. Patients 2 and 3 described significant hematuria, whereas patient 1 had only mild hematuria. Flank pain was also an initial complaint in patients 1 and 3. Pyuria was noted in patient 3 at presentation, but all urine cultures were negative. None of the patients complained of irritative urinary symptoms. Each case was investigated further with laboratory tests, imaging, and ureteroscopy.

Patients 1 and 2 underwent renal ultrasonography as the initial diagnostic procedure. In both cases hydronephrosis was demonstrated. In the case of patient 1 , an unusualappearing filling defect with associated calcification in the upper pole of the right kidney was noted. Subsequent CT (Fig. 1a) showed this filling defect to be a mixed cystic and solid lesion containing both enhancing soft tissue and calcifications. CT in patient 2 (Fig. 2) confirmed the presence of hydronephrosis. Patient 3 was initially evaluated with an intravenous urogram, which revealed hydroureteronephrosis. After imaging, all three patients underwent ureteroscopy.

In patient 1 , ureteroscopy demonstrated calcified, fibrotic tissue located primarily in one wall of the upper pole calix. Ureteroscopy in patient 2 revealed an irregular proximal left ureter that appeared friable but without a mass for approximately $6 \mathrm{~cm}$ of the ureteral length. The ureteroscopy was similar in patient 3 , demonstrating ragged and friable mucosa without a discrete mass, limited to the distal ureter. Biopsies were obtained from all three patients using ureteroscopic cup forceps. Once enough tissue had been obtained, which required several passes of the forceps, the biopsies were submitted in formalin for standard processing. Biopsies in all three patients contained amorphous material that displayed apple green birefringence under polarized light when stained with Congo red stain, indicative of amyloidosis. No malignancy was evident in any of the biopsies. We also obtained saline barbotage through the ureteroscope in each case, and all were negative for neoplasm.

We recommended a conservative treatment approach in all three cases. Patients 1 and 2 were managed with surveillance imaging and routine follow-up appointments, without any medical or surgical interventions. Patient 3 was managed initially with an internal ureteral stent, and subsequently underwent distal ureterectomy and Boari flap ureteroneocystostomy, which was performed without complications. None of the patients received medical treatment for their amyloidosis.

Patient 1 has been closely monitored with CT at 3 months, 9 months, and then annually. Representative CT images, revealing a stable lesion and a normal-appearing collecting system, are shown in Figure $1 \mathrm{~b}$ and c. Clinically, the patient has not experienced any additional episodes of hematuria or

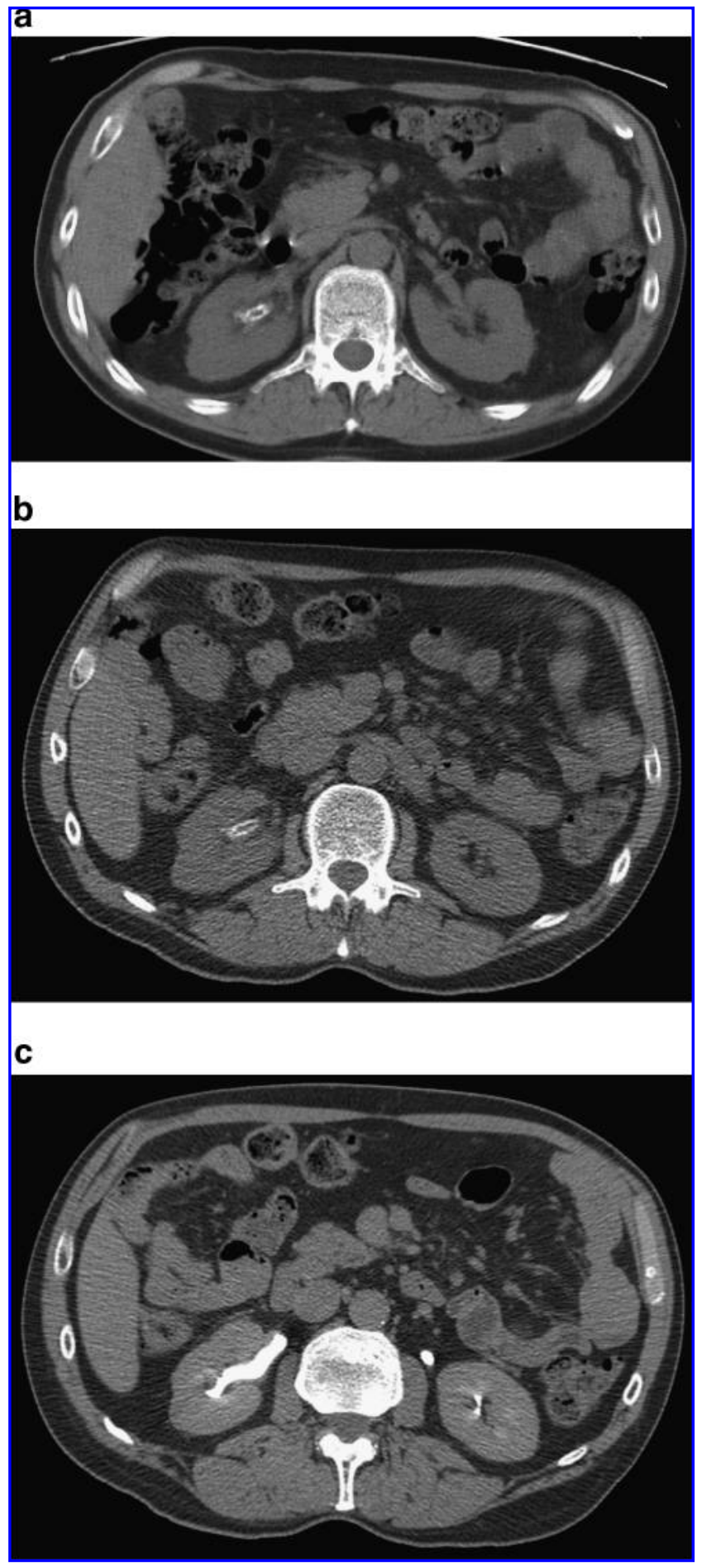

FIG. 1. (a) Patient 1, noncontrast CT at presentation showing calcification of the right renal pelvis. (b) Patient 1, four-year follow-up noncontrast CT showing stable calcification of the right renal pelvis. (c) Patient 1, four-year followup CT with intravenous contrast showing no dilation of the right renal collecting system.

flank pain. Follow-up from ureteroscopy until last imaging is 6 years. Patient 2 reports no flank pain and no additional episodes of hematuria. A renal ultrasonography 15 months after diagnosis reveals persistent moderate left 


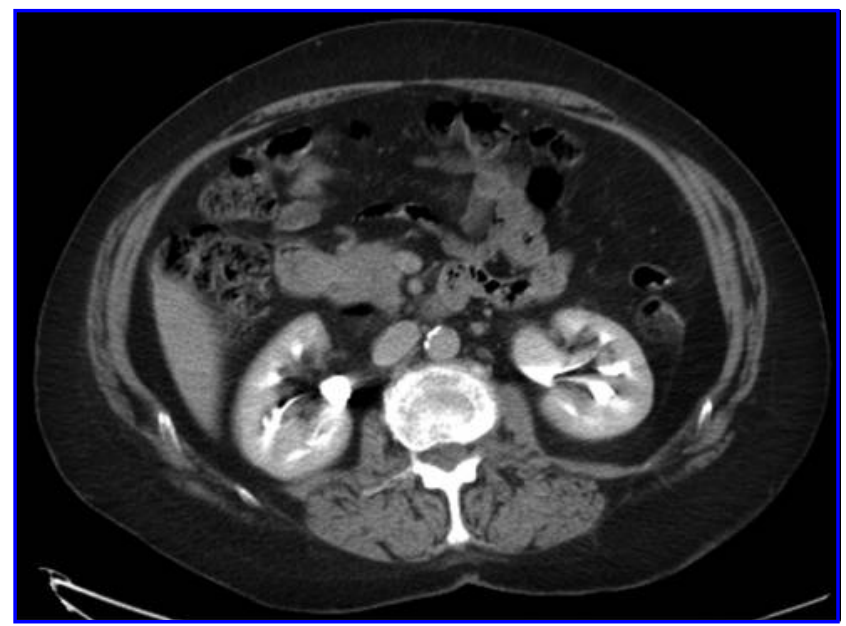

FIG. 2. Patient 2, CT at presentation showing left hydronephrosis.

hydroureteronephrosis but no parenchymal loss. Cystoscopy performed at that time demonstrated clear efflux of urine bilaterally, suggesting against obstruction. Patient 3 underwent intravenous urography at 6 weeks and 1 year postoperatively, both of which demonstrated good function and drainage of the left kidney. She has subsequently been followed with regular office visits and imaging. Magnetic resonance imaging (Fig. 3) obtained at 6 years showed complete resolution of the hydroureteronephrosis. She has had one episode of pyelonephritis, but otherwise has had an unremarkable course in the 8 years since ureteroneocystostomy. With follow-up of 15 months, 6 years, and 8 years, none of the patients have displayed clinical or radiographic signs of progressive disease.

\section{Discussion}

Amyloidosis refers to a group of diseases characterized by the extracellular deposition of amorphous, insoluble, proteinaceous material. Of the four major forms (primary, secondary, heredofamilial, and $\beta_{2}$-microglobulin-associated), the two most frequently encountered forms are primary and second-

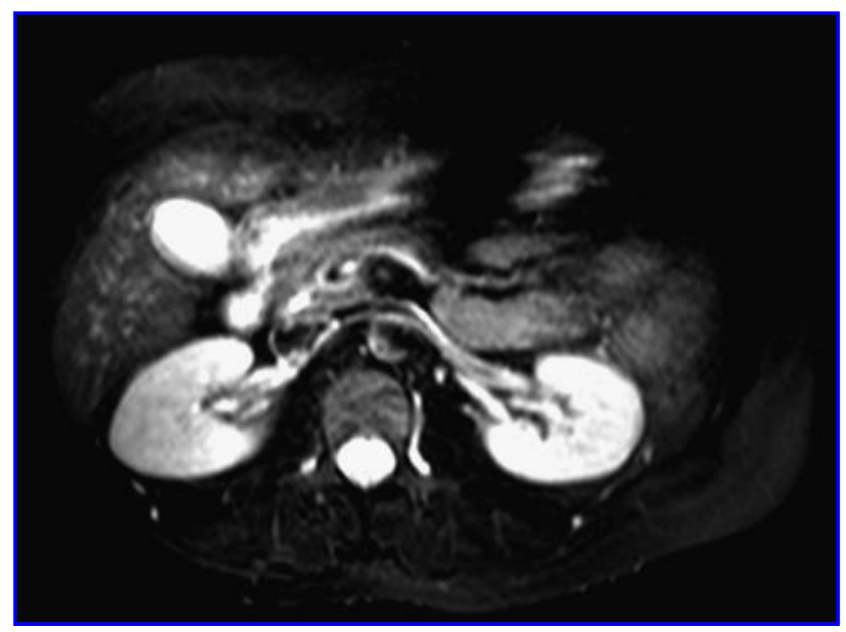

FIG. 3. Patient 3, six-year follow-up magnetic resonance imaging showing no abnormalities in the left renal collecting system. ary. Primary amyloidosis is usually seen in association with an underlying immune dyscrasia, such as multiple myeloma and Waldenström's macroglobulinemia. The deposited fibrils are derived from monoclonal light chains with the $\lambda$ chain seen in higher frequency. ${ }^{2,3}$ In secondary amyloidosis, the deposited material is derived from the acute phase reactant, serum amyloid A protein, and is associated with a chronic inflammatory state such as rheumatoid arthritis, ankylosing spondylitis, and inflammatory bowel disease. ${ }^{4}$ Heredofamilial amyloidosis is the least common and arises as a result of an autosomal dominant single-point mutation in the gene coding for the transthyretin protein. $\beta_{2} \mathrm{M}$-Associated amyloidosis is seen almost exclusively in patients on long-standing hemodialysis.

Amyloidosis also can be classified into systemic or localized, based on its pattern of organ deposition. The majority of cases are systemic, involving multiple organ systems. Systemic amyloidosis is usually primary, progressive, and fatal. ${ }^{1}$ Localized amyloidosis occurs much less frequently than systemic amyloidosis, and involves a single-organ system. The respiratory tract, skin, and genitourinary tract are the most commonly involved. In the urinary tract, localized amyloidosis has been described in the renal pelvis, ${ }^{5}$ unilateral $^{6}$ and bilateral ${ }^{7}$ ureters, bladder, ${ }^{8}$ and urethra. ${ }^{9}$ The bladder is the most affected site. ${ }^{2}$ The majority of reported cases of localized urinary tract amyloidosis are of the primary type, but secondary localized amyloidosis has been reported in the absence of a chronic systemic inflammatory state. ${ }^{10}$ The pathogenesis of primary localized amyloidosis remains undetermined to date but has been postulated to be caused by chronic and recurrent inflammation with migration of lymphoplasmic cells and subsequent aberrant monoclonal proliferation and local secretion of light chains. These light chains, which are transformed to amyloid by lysosomal degradation, are deposited in the affected tissue(s). . $^{1,3}$

Primary localized amyloidosis of the urethra and bladder is a relatively rare condition but is of interest because its clinical presentation and radiographic and cystoscopic appearance mimic that of urothelial cell carcinoma. Amyloidosis of the upper urinary tract shares these characteristics, but is an even more seldom-encountered entity. The typical presentation of urinary tract amyloidosis, regardless of its location, is gross painless hematuria, ${ }^{2}$ and all of our patients presented with this as an initial sign. Two of the patients also complained of flank pain, and all had hydronephrosis at the time of presentation. These features of ureteral obstruction are consistent with the reported literature on upper tract amyloidosis ${ }^{6-9,11,12}$ and, of course, can also be seen with neoplasm. Severe irritative symptoms are common in patients with bladder amyloidosis, ${ }^{2,3}$ but these were characteristically absent in our three patients with upper urinary tract amyloidosis. Another difference between upper and lower urinary tract amyloidosis is that bladder amyloidosis is diffuse or present at multiple discrete sites in most patients, ${ }^{2}$ whereas in our three upper tract amyloidosis patients only a single lesion was noted (although multiple sites of involvement of the ureter have been reported $\left.^{13}\right)$.

Given its presentation, the majority of cases of urinary tract amyloidosis are initially misdiagnosed as malignancy. In most cases bladder lesions are ultimately accurately identified as amyloidosis before a cystectomy is performed, ${ }^{2,3}$ but in the majority of upper tract amyloidosis cases the correct diagnosis is not made until after nephroureterectomy. ${ }^{7,9,14,15}$ All of our 
patients were spared nephroureterectomy owing to our usual practice of ureteroscopic biopsy before nephroureterectomy (in the absence of cytologies positive for urothelial carcinoma).

Various methods have been employed in treating urinary tract amyloidosis. For bladder lesions electrocautery or laser fulguration, transurethral resection and partial cystectomy have all been employed based on the size and number of lesions. ${ }^{2,3}$ Medical management with intravesical dimethyl sulfoxide, oral colchicine, and cepharanthine has been reported in rare cases with limited success. ${ }^{16-18}$ Nephroureterectomy is the predominant treatment for upper urinary tract amyloidosis, but local resection with reimplantation has been reported. $^{7,19}$ There are no reported cases of any successful medical interventions in the treatment of upper tract amyloidosis.

Of our three patients, one was treated with distal ureterectomy and Boari flap ureteroneocystostomy to address the ureteral obstruction, but the other two received no immediate intervention and have remained without signs or symptoms of ureteral obstruction or progressive amyloidosis. The latter two patients are the first reported cases of upper urinary tract amyloidosis in which no therapeutic intervention has been taken and the patients have been merely monitored with imaging. None of our patients developed any additional lesions or had recurrence of their symptoms nor did any progress to systemic disease.

Our study demonstrates that upper urinary tract amyloidosis shares many commonalities with bladder amyloidosis but also has some unique features that may help guide diagnosis, treatment, and follow-up. Once systemic disease has been ruled out by urinary Bence-Jones protein, 24-hour urine protein electrophoresis, and serum protein electrophoresis-and in the absence of significant obstruction-an upper urinary tract lesion does not need to be excised or ablated. Further, serial imaging can be used to monitor the progression of the lesion instead of repeat ureteroscopy.

\section{Conclusions}

Primary localized amyloidosis of the upper urinary tract is a rare condition but is of interest because of its clinical resemblance to urothelial carcinoma. Accurate diagnosis can be made by Congo red staining of an ureteroscopic biopsy sample, which can avoid unnecessary nephroureterectomy. Early eradication is not necessary with nonobstructive localized amyloidosis of the upper urinary tract. Rather, regular follow-up with serial imaging can be used to monitor the progression of the lesion. Ablation or local excision need be employed only if the lesion becomes obstructive or otherwise problematic.

\section{Disclosure Statement}

Tudor Borza, B.S.-none.

Rajal B. Shah, M.D.-none.

Gary J. Faerber, M.D.-none.

J. Stuart Wolf, Jr., M.D.-Terumo Corporation (consultant), Gyrus-ACMI (lecturer).

\section{References}

1. Glenner GG. Amyloid deposits and amyloidosis. The betafibrilloses (parts I and II). N Engl J Med 1980;302:1283-1292, 1333-1343.
2. Tirzaman O, Wahner-Roedler DL, Malek RS, et al. Primary localized amyloidosis of the urinary bladder: A case series of 31 patients. Mayo Clin Proc 2000;75:1264-1268.

3. Merrimen JLO, Alkhudair WK, Gupta R. Localized amyloidosis of the urinary tract: Case series of nine patients. J Urol 2006;67:904-909.

4. Lance NJ, Curran JJ. Amyloidosis in a case of ankylosing spondylitis with a review of the literature. J Rheumatol 1991; 18:100-103.

5. Iida S, Chujyo T, Nakata $Y$, Iuchi $H$. A case of amyloidosis of the renal pelvis. Acta Urol Jpn 2003;49:423-426.

6. Fugita OE, DeLatorre CG, Kavoussi LR. Primary localized amyloidosis of the ureter. J Urol 2001;58:281.

7. Mullin EM, Trostle DR, Fetzer AE, et al. Bilateral amyloidosis of the ureter associated with carcinoma. J Urol 1984;132: 1181-1183.

8. Thomas SD, Sanders PW, Pollack H. Primary amyloidosis of urinary bladder and ureter, cause of mural calcification. J Urol 1977;9:586-589.

9. Mark IR, Goodland J, Lloyd-Davies RW. Localized amyloidosis of the genitourinary tract. I R Soc Med 1995;88: 320-324.

10. Akram CM, Al-Marhoon MS, Mathew J, et al. Primary localized AA type amyloidosis of urinary bladder, case report of rare cause of episodic painless hematuria. J Urol 2006;68: 1343.e15-7.

11. Paraskevas KI, Anagnostou D, Bouris C. Anurea caused by primary amyloidosis of the lower third of the ureters, ureterovesicular junction and the urinary bladder. Int Urol Nephrol 2004;36:339-342.

12. Duffau $P$, Imbert $Y$, De Faucal $P$, et al. Primary localized amyloidosis of the urinary tract, a case series of five patients. La Rev Med Interne 2005;26:288-293.

13. Cuckow PM, Bullock KN. Multifocal ureteric amyloidosis. Br J Urol 1991;68:207-208.

14. Awakura Y, Mizutani Y, Kakehi Y, et al. A case of localized amyloidosis of the ureter. Acta Urol Jpn 1996;42:135-138.

15. Kitsukawa S, Hosoda S, Otsuru N, Matsumoto T, Yamamoto Y, Matsumoto T. Localized amyloidosis of the ureter: A case report. Acta Urol Jpn 2006;52:131-134.

16. Malek RS, Wahner-Roedler DL, Gertz MA, et al. Primary localized amyloidosis of the bladder: Experience with dimethyl sulfoxide therapy. J Urol 2002;168:1018-1020.

17. Livingstone RR, Sarembock LA, Barnes RD, et al. Colchicine therapy in primary amyloidosis of the bladder: A case report. J Urol 1989;142:1570-1571.

18. Nishiyama T, Gejyo F, Katayama Y, et al. Primary localized amyloidosis of the bladder: A case of AL (lambda) amyloid protein and combination therapy using dimethyl sulfoxide and cepharanthine. Urol Int 1992;48:228-231.

19. Fernandez $H$, Rovegno $A R$, Marrugar $R$, et al. Primary ureteral amyloidosis. Arch Esp Urol 1998;51:485-490.

Address correspondence to: J. Stuart Wolf, Jr., M.D. Department of Urology University of Michigan Health System 1500 East Medical Center Drive, TC 3875 Ann Arbor, MI 48109-5330

E-mail:wolfs@umich.edu

\begin{tabular}{|c|}
\hline Abbreviation Used \\
$\mathrm{CT}=$ computed tomography
\end{tabular}

